\title{
Long integral temperature Brillouin sensor for off- shore wind energy power supply lines
}

\author{
M. A. Quintela ${ }^{a}$, A. Ullán ${ }^{\mathrm{a}}$, A. Quintela ${ }^{\mathrm{a}}$, C. Galindez ${ }^{\mathrm{a}}$, R. A. Perez-Herrera ${ }^{\mathrm{b}}$, M. López- \\ $\mathrm{Amo}^{\mathrm{b}}$ and J. M. Lopez-Higuera ${ }^{\mathrm{a}}$ \\ ${ }^{a}$ Photonics Engineering Group, Universidad de Cantabria, Avda. Castros S/N, 39005 Santander, Spain \\ ${ }^{\mathrm{b}}$ Dpto. Ingeniería Eléctrica y Electrónica, Universidad Pública de Navarra, Campus de Arrosadia s/n, 31006 \\ Pamplona, Spain
}

\begin{abstract}
A hybrid Erbium-Brillouin fiber laser sensor to measure the temperature along $22 \mathrm{~km}$ fiber is proposed and experimentally demonstrated. A multi-line laser oscillation is induced by the Brillouin gain of different concatenated transducer fiber sections placed in the ring cavity. Integral temperature measurements of each fiber section are obtained through each laser line. This sensor can be used to monitor the temperature of off-shore wind energy power cables.
\end{abstract}

Keyword list: Optical fiber sensors, Brillouin scattering, Erbium fiber laser

\section{INTRODUCTION}

The introduction of off-shore wind farms has implied a revolution in the area of wind electricity generation[1]. Large wind farms are currently under development from land to coastal waters because of the rich wind marine resources, scarcely occupied land and minor environmental constraints. An off-shore wind farm is typically constructed 10-40 km away from the coast[2]. The electricity produced by wind turbine must be transmitted to the on-shore consumers through the submarine cables. One of the most important issues in the design of a submarine transmission is the choice of the cable type. The type of cable directly affects the cost of the system and its installation. Moreover, the submarine transmission must be reliable and economical for a minimum of 25 or 30 years due to the expensive investment.

The optimum distribution of the electricity (in the specific duration and the operation of the cable) is directly related to the cable temperature[3]. An important factor to be taken into account is the maximum operation temperature. This factor can be exceeded mainly for: poor feasibility studies, choosing design parameters closer to the maximum capacity of the cable and rapid growth in user demands. Another important factor is the presence of "hot areas". The operation time period can be seriously reduced if these "hot areas" are not detected or neglected. Therefore, the temperature must be monitored along the cable.

Optical fiber temperature monitoring has been used with underground cable for more than a decade. Currently, these sensing systems are also starting to be applied in submarine cable systems. Today, most submarine cables integrate multiple single-mode optical fibers for data transmission and several spare fibers for others purposes, for example strain and/or temperature monitoring. On the other hand, the optical fiber can be replaced by with new ones when it is required, given that the optical fiber integration in the cable does not depend on productions and implementation processes of the cable. This will permit the optimization of the fiber channel, for example employing different type of fibers to measure the temperature along several tens of kilometers.

In this work, an integral temperature sensor based on a hybrid Erbium-Brillouin ring fiber laser to measure temperature in multiple transducer sections along $22 \mathrm{~km}$ off- shore wind energy power cables is proposed and experimentally demonstrated.

\section{SET-UP AND PRINCIPLE OF OPERATION}

The experimental setup of the proposed Erbium-Brillouin ring fiber laser sensor configuration is shown in Fig. 1. The fiber cavity is constituted by a highly erbium-doped fiber (EDF) together with $22 \mathrm{~km}$ of the fiber transducer. The EDF used is Er-30 by Liekki, which shows an absorption coefficient of $30 \mathrm{~dB} / \mathrm{m}$ at $\lambda=1530 \mathrm{~nm}$ and has a length of $7 \mathrm{~m}$.

21st International Conference on Optical Fiber Sensors, edited by Wojtek J. Bock, Jacques Albert, Xiaoyi Bao, Proc. of SPIE Vol. 7753,775324 - (C) 2011 SPIE · CCC code: 0277-786X/11/\$18 · doi: 10.1117/12.885887 


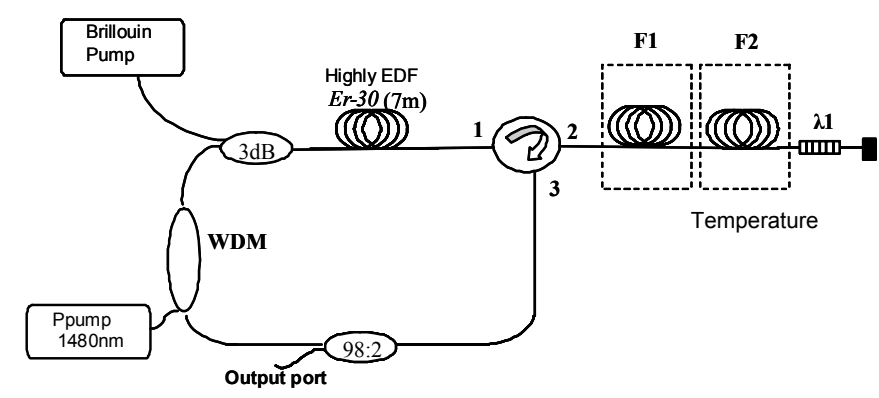

Fig. 1. Experimental setup of Erbium-Brillouin ring fiber laser sensor configuration.

The fiber transducer consists of $\mathrm{N}$ concatenated sections of different fibers. To demonstrate the system we used two spools of fiber (F1 and F2), with lengths of $15 \mathrm{~km}$ and $7 \mathrm{~km}$, respectively coiled without mechanical strain. F1 is a Sumitomo single mode fiber and F2 is an Alcatel TERALIGHT single mode fiber.

A laser at $1480 \mathrm{~nm}$ was used as the pump source for the EDF and a 1480/1550-nm wavelength-division multiplexer (WDM) was employed to multiplex the pump signal and the oscillating signals. This configuration also includes a 3-dB coupler to incorporate the Brillouin pump (BP) signal, provided by a laser diode, into the laser cavity. The optical circulator was used to guide the propagation of the BP signal into the two spools of single mode fiber (Brillouin gain medium) and to insert the Brillouin Stokes (BS) signal into the ring, ensuring the unidirectional propagation. A fiber Bragg grating (FBG) was used to reflect the BP and BS signals back into the Brillouin gain medium, thus reducing the Brillouin threshold. A $98 \%$ coupler is used to extract $2 \%$ of the laser output power from the ring. All free terminations of the system have been immersed in refractive-index-matching gel to avoid undesired reflections. The Brillouin pump signal and the Brillouin Stokes signals (one per spool of fiber) are measured using a high-resolution optical spectrum analyzer (BOSA-C Aragon Photonics) which offers simultaneously a high resolution $(0.08 \mathrm{pm})$ and a high dynamic range $(>80 \mathrm{~dB})$.

In this laser ring configuration, the injected BP signal will be first amplified by the EDF. It will be then guided into the Brillouin gain medium by the optical circulator. When the BP power exceeds the threshold power of the Brillouin gain medium, stimulated Brillouin scattering will appear and thus the BP signal will create the first order Stokes signal which will propagate in the opposite direction to the one of the BP signal. The first order Stokes signal will be inserted again into the laser cavity by the optical circulator. It will be amplified by the EDF and it will follow the same progression as the BP signal. When it exceeds the Brillouin threshold condition of the Brillouin gain medium, it will create then the second order Stokes signal. In the same way, the second order Stokes signal will create the third order Stokes signal. The process will continue until the next higher order Stokes signal power becomes too small to exceed the threshold condition.

The mechanism of this sensor is based on the frequency positioning of the BS signal. The BS frequency shifts with respect to the BP frequency a value equal to the Brillouin frequency shift. This shift is given by $v_{B}=2 n V_{a} / \lambda_{o}$, where $n$ is the fiber effective refractive index, $\lambda_{0}$ the vacuum wavelength of the pump light and $V_{a}$ the acoustic velocity in the fiber core. The parameters $\mathrm{n}$ and $\mathrm{V}_{\mathrm{a}}$ are dependent of the fiber structure and composition; and also on the temperature and strain of the optical fiber[4]. By determining the central frequency of the Brillouin scattered light of each spool of fiber, temperature changes in each transducer section can be measured independently. The Brillouin frequency shift has a linear dependence on temperature.

\section{RESULTS}

Two fundamental aspects in the design of the hybrid Erbium-Brillouin ring laser have to be explored in order to achieve an optimized cost-effective temperature laser sensor. The first challenge is to obtain a high stability in Brillouin-Erbium 
fiber laser suppressing unstable modes (self-lasing cavity modes) oscillating in this cavity. These unwanted oscillating modes share the same gain medium with Brillouin Stokes lines and therefore the gain competition occurs. To solve this problem a FBG has been used as band-pass filter showing a Bragg wavelength that coincides with the BP wavelength. Thus, the amplification band is delimited. This technique does not limit the operation range of the BP wavelength in the laser sensor specifications as occurs with other techniques where the BP wavelength must be chosen within the peak gain of the laser cavity[5]. This implies that, when the BP signal is detuned away from the peak gain, the BP signal must have adequate power to suppress the unstable modes.

Another challenge is to induce the oscillation of only the first-order Stokes signal of each transducer fiber section with a very low BP power. The threshold BP power as a function of the EDF pump power was measured. The experimental results are shown in Fig. 2(a). These experimental results were carried out for a BP wavelength of $1550.7 \mathrm{~nm}$. The threshold power is defined as the power at which the steady first order Stokes signal is larger than $10 \mathrm{~dB}$. As can be shown the threshold BP power increases with the EDF pump power. The number of Stokes signals (output channels) as a function of the BP power was also measured. The experimental results for an EDF pump power of $27 \mathrm{dBm}$ and a BP wavelength of $1550.7 \mathrm{~nm}$ are shown in Fig. 2(b). As can be clearly seen in this figure, for a fixed EDF pump power, a higher BP power corresponds to a reduced number of output channels, given that the higher the BP power is, the higher is the EDF pump power necessary to achieve the oscillation, which is in agreement with the threshold curve showed in Fig. 2(a).It can be deduced that when the BP power is low the oscillation of only the first-order Stokes signal is not possible. This problem can be solved using a FBG with a full-width at half-maximum (FWHM) that permits filter only the first channel and eliminate the other channels when the BP power is very low. For the fibers used in this work (F1 and F2), where $v_{\mathrm{B}}$ are $10.6 \mathrm{GHz}(0.085 \mathrm{~nm})$ and $11.07 \mathrm{GHz}(0.089 \mathrm{~nm})$, respectively, an adequate value for the FWHM of the FBG is $0.15 \mathrm{~nm}$.
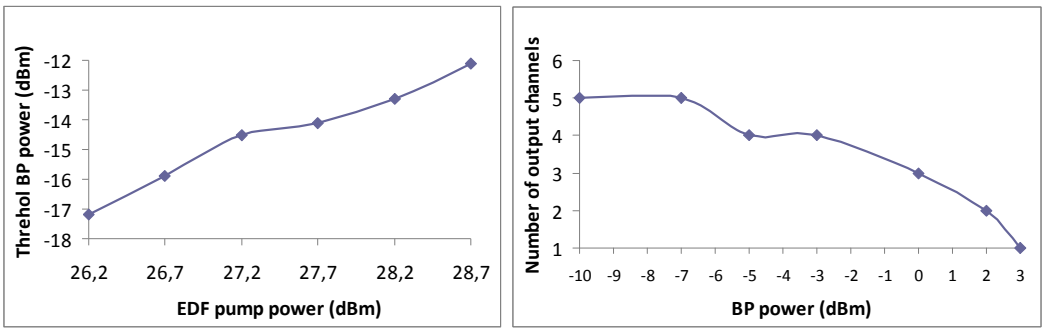

Fig. 2. (a) Threshold BP power versus the EDF pump power. (b) Number of output channels versus the threshold BP power.

The output optical spectrum of the hybrid Erbium-Brillouin ring fiber laser for an EDF pump power of $27 \mathrm{dBm}$, a BP power of $-10 \mathrm{dBm}$ and a FBG with a FWHM of $0.4 \mathrm{~nm}$ is shown in Fig. 3(a). As can be shown in Fig. 3(a), the first and second order Stokes signals of the two different fiber spools together with the BP signal achieve the oscillation simultaneously. For each fiber section, the Stokes signals are equally spaced, separated by $10.6 \mathrm{GHz}$ for F1 and 11.7 $\mathrm{GHz}$ for F2. The output optical spectrum of this laser for the same input powers and a FBG with a FWHM of $0.15 \mathrm{~nm}$ is shown in Fig. 3(b). As can be seen, only three laser lines (the first-order Stokes signals of F1 and F2, and the BP signal) are oscillating. From this figure, we point out that the narrow FWHM linewidth is approximately $6 \mathrm{MHz}$. This makes it possible to measure very small temperature variations.

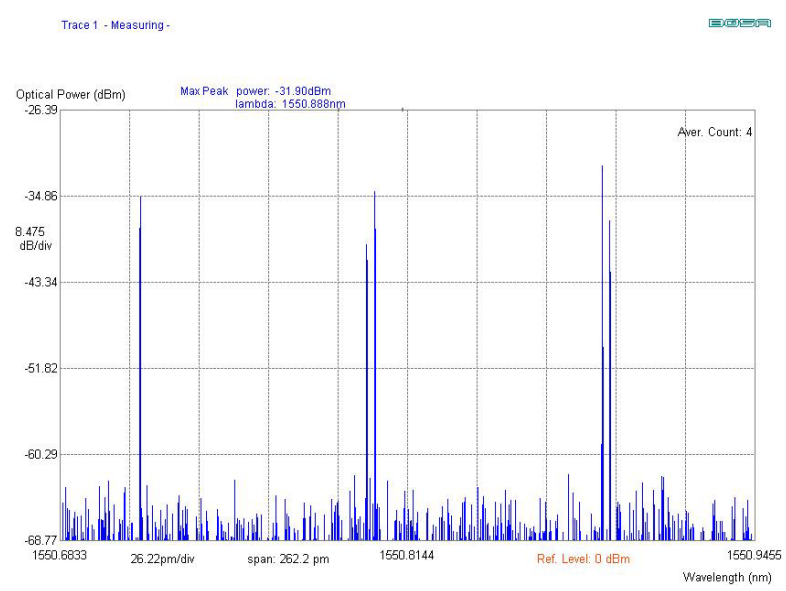

Fig. 3(a) Output optical spectrum measured by the BOSA for a FBG at $\lambda=1550.7 \mathrm{~nm}$ with a FWHM of $0.4 \mathrm{~nm}$. 


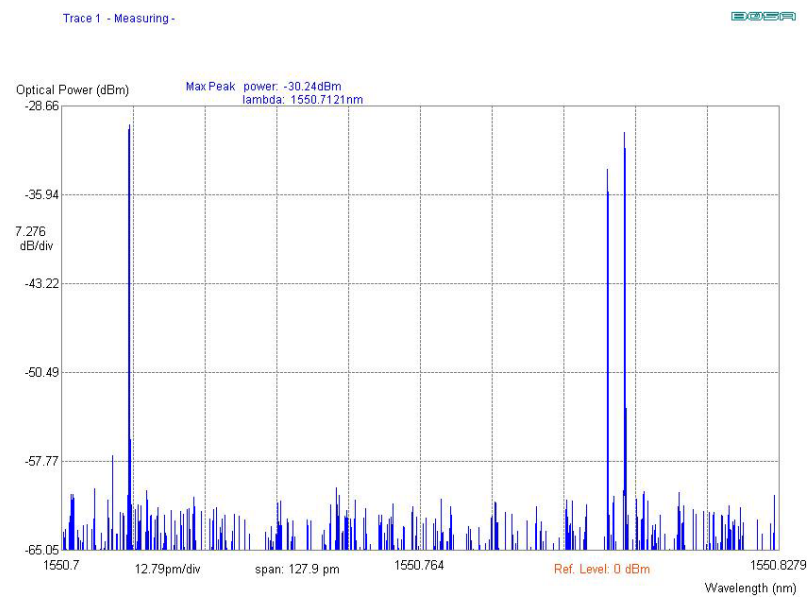

Fig. 3(b). Output optical spectrum measured by the BOSA for a FBG at $\lambda=1550.7 \mathrm{~nm}$ with a FWHM of $0.15 \mathrm{~nm}$.

Temperature measurements of each fiber section are obtained through the variation in each Brillouin laser signal. The data for the variations in the Brillouin laser signal with the temperature are similar to the ones presented in [6]. Those present a linear dependence with the temperature of about $1.1 \mathrm{MHz} /{ }^{\circ} \mathrm{C}$. The measurement range of temperature is delimited by the gap between Brillouin signals, i.e. the characteristic Brillouin shift into the fibers. The number of fiber spools depends on the fiber length, the Brillouin thresholds, the Brillouin gain coefficient of each fiber and the pump powers. Furthermore, the temperature measurement can be performed in real time through a heterodyne detection scheme as proposed in [6].

\section{CONCLUSIONS}

In this paper we proposed and experimentally measured a simple hybrid Erbium-Brillouin fiber laser sensor for sensing temperature in multiple transducer concatenated sections along $22 \mathrm{~km}$. Each transducer fiber section generates a laser signal in a different Brillouin wavelength that depends on the fiber properties and temperature. In this laser sensor, the $\mathrm{BP}$ power required to induce the laser signal from the Brillouin gain is very low $(-10 \mathrm{dBm})$. This permits to reduce the cost of the sensor system. Moreover, this technique does not need special optical fibers as transducer, the frequency variations are determined from a strong laser signal and an electro-optic modulator is not used. These results are promising for practical long range Brillouin-based optical sensing systems in off- shore wind energy power cables.

\section{ACKNOWLEDGEMENTS}

The authors would like to acknowledge the support provided by the Spanish CICYT through the project TEC200767987-C02 and TEC2010-20224-C02, European COST-299 action and Liekkis' fibers.

\section{REFERENCES}

[1] Z. Chen, and F. Blaabjerg, "Wind farm-A power source in future power systems," Renewable \& Sustainable Energy Reviews, 13(6-7), 1288-1300 (2009).

[2] Z.X. Wang, C.W. Jiang, Q.A. Ai, and C.M. Wang, "The key technology of offshore wind farm and its new development in China," Renewable \& Sustainable Energy Reviews, 13(1), 205-211 (2009).

[3] G. Yilmaz, and S.E. Karlik, "A distributed optical fiber sensor for temperature detection in power cables," Sensors and Actuators a-Physical, 125(2), 148-155 (2006).

[4] J. Lopez-Higuera, [Handbook of optical fibre sensing technology] John Wiley \& Sons Inc, (2002).

[5] N. Hambali, M.A. Mahdi, M.H. Al-Mansoori, A.F. Abas, and M.I. Saripan, "Investigation on the effect of EDFA location in ring cavity Brillouin-Erbium fiber laser," Optics Express, 17(14), 11768-11775 (2009).

[6] C.A. Galindez, F.J. Madruga, A. Ullan, M. Lopez-Amo, and J.M. Lopez-Higuera, "Multi-zone temperature sensor using a multiwavelength Brillouin fiber ring laser," Proc. SPIE - Int. Soc. Opt. Eng. (USA). 7503, 75030J (4 pp.). 